\title{
On the Measurement of Weak Repulsive and Frictional Colloidal Forces by Reflection Interference Contrast Microscopy
}

\author{
Joachim Rädler* and Erich Sackmann \\ Physik Department (Biophysik E22), TU-München, James-Franck-Strasse, \\ D-8046 Garching, Germany
}

Received October 15, 1991. In Final Form: January 2, 1992

\begin{abstract}
The motion of microscopical spheres close to a surface in a liquid suspension can be traced by reflection interference contrast (RIC) microscopy in combination with real-time image processing. The temporal fluctuations of the absolute sphere-to-substrate distance are determined from changes of interference fringe pattern (Newtonian rings). Both the shape about its minimum and the absolute minimum equilibrium distance of the interaction potential can be obtained by analyzing the distribution of distances in terms of a Boltzmann distribution. The time-autocorrelation function of distances yields the hydrodynamic friction. The method has been applied to the interaction of latex spheres with glass substrates in salt solutions of different ionic strength. The results correspond to classical electrostatic double layer theory that leads to a characteristic dependence of the mean separation distance and the mean square displacement on the radius of the spheres. The hydrodynamic friction close to a wall exhibits the predicted inverse proportionality to the sphere-wall distance. It is demonstrated that the method can be applied to study the interaction between biologically relevant objects such as giant vesicles with bilayer covered substrates.
\end{abstract}

\section{Introduction}

The interaction between cells and the interaction of cells with surfaces is a most complex process owing to the interplay of specific and nonspecific forces. In general, cell adhesion involves specific molecular bonding and even active rearrangement of the cell membrane. However, unspecific interaction forces play a dominant role in adhesion and repulsion. Therefore experimental methods that study colloidal surface interaction are of great importance to biological science.

There are a number of different techniques that allow quantitative measurement of cell-cell interaction. ${ }^{1}$ From the physical point of view, however, better understood is the interaction between model surfaces such as lecithin bilayers that has been widely investigated..$^{2-5}$ In this case forces down to molecular distances can be measured, where additional short range molecular or structural forces come into play. ${ }^{5}$ On the other hand most of the latter techniques cannot be applied to real cells. Moreover since the methods are not dynamic, they neglect hydrodynamic dissipative forces.

Recently Prieve and Alexander ${ }^{6}$ introduced a new method to measure repulsive long range forces between microscopical spheres and an underlying substrate. The interaction potential can be determined from the Brownian motion of the particle by counting the number of times that a certain distance is taken during the random distance fluctuations of the sphere. The potential is simply related to the distribution function of distances by the Boltzmann distribution. The technique has been refined by Prieve and Frej ${ }^{7}$ using total internal reflection microscopy (TIRM) to measure the sphere-substrate distance. However, since in this technique the distance is measured by

(1) Bongrand, Physical basis of cell-cell adhesion; CRC Press: Boca Raton, FL, 1988

(2) Lis, L. J.; McAlister, M.; Fuller, N.; Rand, R. P.; Parsegian, V. A Biophys. J. 1982, 37, 657 .

(3) Vrij, A.; Joosten, J. G. H.; Fijnaut, H. M. Adv. Chem. Phys. 1981, 48,329 .

(4) Evans, E.; Needham, D. J. Phys. Chem. 1987, 91, 4219.

(5) Marra, J.; Israelachvilli, J. Biochemistry 1985, 24, 4608.

(6) Prieve, D. C.; Alexander, B. M. Science 1976, 231, 1269.

(7) Prieve, D. C.; Frej, N. A. Langmuir 1990, 6, 396. scattering of an evanescent field, only relative changes in distance can be measured and therefore only the shape but not the absolute distance dependence of the potential can be determined.

This article is intended to demonstrate that reflection interference contrast (RIC) microscopy on the other hand allows measurements of the absolute separation of a sphere and the underlying surface. With fast image processing, the Brownian motion of a single microsphere can be followed from its interference pattern in real time and its distance to the substrate can be recorded in time intervals of $40 \mathrm{~ms}$. Thus the interaction potential can be determined from the distance distribution function of the particle as suggested by Prieve, and furthermore the dissipative hydrodynamic force can be deduced from the time autocorrelation function of the Brownian motion at the wall.

The method has been applied to polystyrene beads on glass substrate where the interaction potentials were found to correspond accurately to classical DLVO theory. Also the hydrodynamic dissipative force scales with $R^{2}$ as predicted for a sphere close to a wall. Furthermore we measured the interaction of spherical giant vesicles with a supported monolayer of lecithin. This demonstrates that the technique presented in this article has great potential for investigating repulsive cell-substrate interaction in general.

\section{Experimental Method}

The principle of RIC microscopy is depicted in Figure 1. The object is observed by reflection microscopy with epi-illumination with monochromatic light. Then the incident beam $I_{0}$ is partly reflected at the glass-buffer interface $I_{12}$ and the transmitted part is reflected by the object. The interference pattern formed by the superposition of the object beam $I_{23}$ and the reference beam $I_{12}$ is observed by the objective. In the case of perpendicular incidence the reflected intensities are given by the Fresnel equations

$$
I_{i j}=I_{0}\left(\frac{n_{i}-n_{j}}{n_{i}+n_{j}}\right)^{2}
$$

For phase objects the reflected intensities are small and com-

(8) Zilker, A.; Engelhardt, H.; Sackmann, E. J. Phys. (Paris) 48, 2139. 


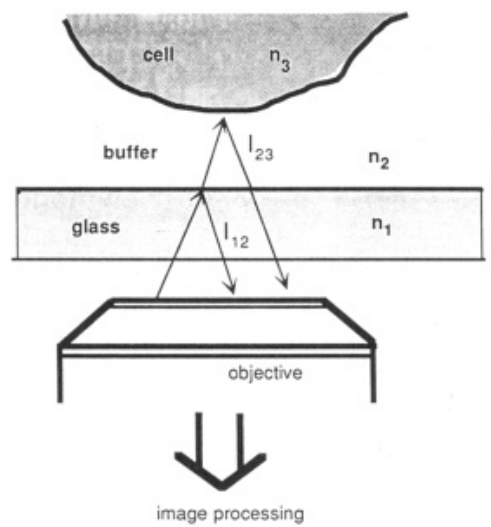

Figure 1. Principle of the reflection interference contrast microscopy. The interference between the object beam $I_{23}$ and the reference beam $I_{12}$ is observed.

parable to the stray light intensity. This problem is overcome by the so-called antiflex technique, ${ }^{9}$ which allows the stray light to be supressed sufficiently. In this technique polarized light is transformed into circular polarized light by a $\lambda / 4$ plate that is situated in the top of the microscope objective. The reflected light passing again through the $\lambda / 4$ plate will be transformed back into polarized light with the polarization rotated by $90^{\circ}$ with regard to the incoming beam. Thus the light, reflected from the object, will pass a second crossed polarizer, while stray reflections from intermediate lenses will not.

The RIC microscopy yields interference pattern that are analogous to Newton rings of spherical objects. The intensity is of the form

$$
I(x)=I_{12}+I_{23}+2\left(I_{12} I_{23}\right)^{1 / 2} \cos \left[\frac{4 \pi n_{2} g(x)}{\lambda}+\delta\right]
$$

where $n_{2}$ denotes the refractive index of the buffer medium and $g(x)$ the separation between the glass substrate and the object. $\delta$ describes the phase shift that occurs at the buffer-object interface. In general the object will have a higher refractive index than the buffer medium and therefore $\delta$ is equal to $\pi$. In the case of multilamellar systems or very thin objects like a single bilayer, the intensity $I_{23}$ has to be calculated with respect to all optical interfaces in the object. Here interference within the layer structure can lead to an effective phase shift $\delta$ at the object that is unequal to $\pi$ or 0 .

Consider now the interference pattern of a single sphere separated from the glass substrate by a distance $h$. Figure 2c shows a typical radial intensity profile received from a latex sphere with a $16-\mu \mathrm{m}$ radius. The radial interference intensity $I(x)$ is described by

$I(x)=A_{0}+A_{1} e^{-b_{1} x^{2}}+A_{2} e^{-b_{2} x^{2}} \cos \left[\frac{4 \pi n_{2}}{\lambda}[g(x)+h]+\delta\right]$

where $A_{2}$ and $b_{2}$ are parameters that fit the decaying contrast of the higher order extrema and $A_{1}$ and $b_{1}$ fit the slightly bent background that arises from diffuse reflection of the upper part of the sphere. The parameters $A_{i}$ and $b_{i}$ are necessary to fit the envelope of the oscillating intensity and are of no further meaning here. The term in brackets determines the phase. Here $h$ is the minimum separation distance between the sphere and the glass surface and $g(x)$ describes the contour of the sphere

$$
g(x)=R-\left(R^{2}-x^{2}\right)^{1 / 2}
$$

Note that the phase of the cosine in eq 3 is determined by only one free parameter, the distance $h$. The radius $R$ of the sphere can be measured separately using dia-illumination in the same experimental setup. Therefore the minimum distance $h$ is obtained by fitting the observed intensity by eq 3 . In Figure $2 c$ an example of a latex bead in $2 \mathrm{mM} \mathrm{NaCl}$ is given that shows also the best fit of the intensity data over the entire range.

In order to observe the temporal fluctuations of the objectsubstrate distance by image processing in real time, a slightly

(9) Patzelt, W. I. Leitz-Mitt. Wiss. Tech. 1979, 7, 141.
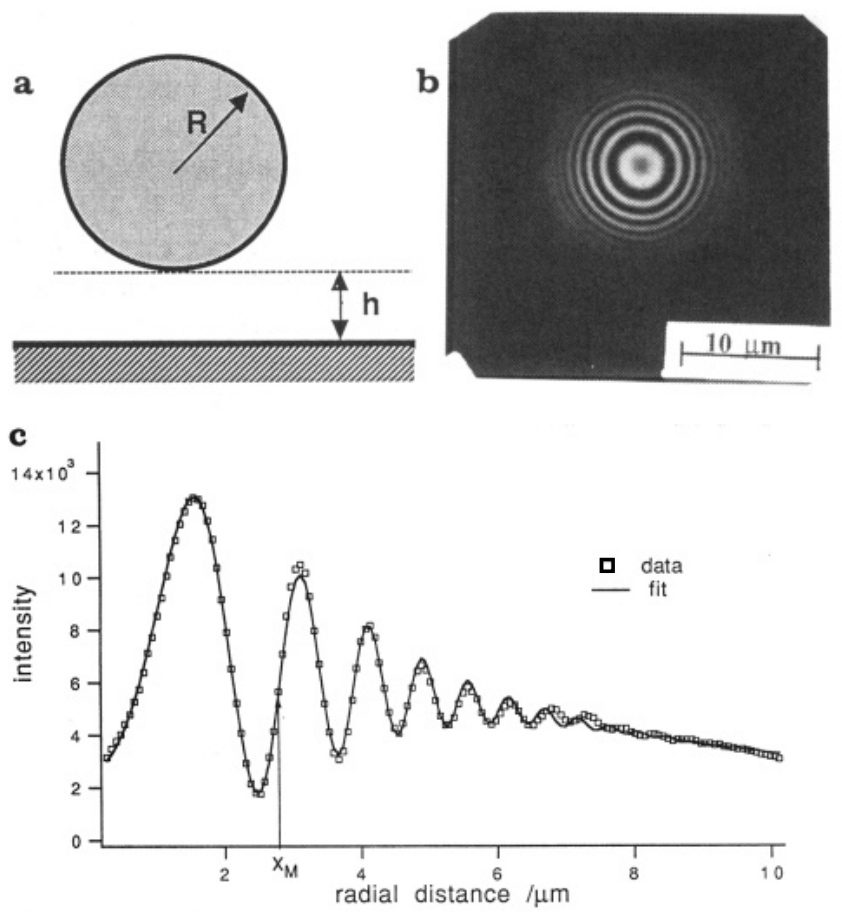

Figure 2. (a) Schematic view of sphere interacting with substrate. The particle can be considered as a Brownian particle trapped in a "wall potential". (b) Interference pattern of a latex sphere of $16 \mu \mathrm{m}$ diameter (Newton rings). The center of the fringe pattern is traced by image processing in real time. (c) Radial intensity corresponding to Figure $2 \mathrm{~b}$. Changes in the spheresubstrate distance $h$ lead to a phase shift of the pattern. The intensity at a fixed position $x_{\mathrm{m}}$ was recorded.

different algorithm is adopted. The program first searches the center of the interference rings and determines the radial intensity $I(x)$. This procedure is applied once for each sphere studied. Subsequently for each frame of $40 \mathrm{~ms}$ the program corrects the position of the center as the sphere moves laterally and measures the intensity at a fixed radius $x_{\mathrm{m}}$ that is chosen to lie in the linear regime between the first minimum and the second maximum (see arrow in Figure 2c). Since the intensities of the neighboring minimum and maximum are known, the change in distance can be determined directly from $I\left(x_{\mathrm{m}}\right)$. The intensity $I\left(x_{\mathrm{m}}\right)$ is averaged over roughly 100 pixels. In this way the relative change in distance can be measured to an accuracy of $1 \mathrm{~nm}$. The determination of the absolute distance $h$ is limited by 2 factors: the numerical aperture of the illumination and the focus which both affect the interference pattern slightly. The effect of the illuminating numerical aperture has been studied by Gingell and Todd. ${ }^{10}$ In agreement with our observations they report that for small illumination numerical aperture (INA $=0.42$ ) and thick objects the agreement with the normal incident calculation is very good. From repeated experiments on one single sphere, we estimate $h$ to be accurate within $4 \mathrm{~nm}$.

\section{Measurements}

The experimental setup has been described in detail by Zilker et al. ${ }^{8}$ The RIC micrographs are taken with a Zeiss Axiomat inverted microscope equipped with an antiflex oil immersion objective (Antiflex Neofluar 63/1.25). Illumination is performed with a high-pressure $\mathrm{Hg}$ lamp (Osram HBO $100 \mathrm{~W}$ ) and monochromatic light of $\lambda=546$ $\mathrm{nm}$ is produced by a combination of an interference and a cutoff filter (Schott A3-546 and OG 530). The whole experimental setup is suspended from the ceiling to prevent impairment from external noise.

Polystyrene latex beads $(R=5-25 \mu \mathrm{m})$ have been purchased from Sigma. The microspheres were dispersed in various concentrations of $\mathrm{NaCl}$ and filled in a measuring

(10) Gingell, D.; Todd, I. Biophys. J. 1979, 26, 507. 
chamber that was made up of two glass coverslips separated by a frame made of $0.15 \mathrm{~mm}$ thick Teflon foil and sealed with silicone grease. The small width of the intermediate gap allows for observation without disturbance by convection. This is demonstrated by the absence of lateral drift.

Image of the interference pattern are taken with a CCD camera (HR 480, Aqua TV, Kempten, FRG), at a rate of 25 frames per second. A frame comprises $604 \times 588$ pixel elements. The magnification of the image onto the camera can be changed by an intermediate optical system of lenses in the microscope. It is chosen large enough to ensure sufficient averaging over roughly 100 pixels for the second maximum of the interference rings. The image processing is carried out on a Datacube system (Datacube Peabody, Boston) equipped with a 68000 Motorola processor and MaxVideo sofware (Datacube). The images are digitized into 256 gray levels. The image processing runs in real time and performs the steps as described above. In the case of latex spheres, measurements are typically performed over a period of $5 \mathrm{~min}$ allowing a sequence of 7500 intensities to be taken corresponding to a sequence of 7500 heights $h$. The autocorrelation $\langle d h(t) d h(0)\rangle$ and the distribution function $P(h)$ of the distances are calculated by the program from the stored sequence of $I\left(x_{m}\right)$ values.

\section{Theory}

Consider a sphere of radius $R$ which is repelled by a flat surface and is therefore found at a position $h$ above the surface, where gravitational and repulsive forces balance (Figure 2a). Let $h$ be the smallest distance between the sphere and the plane. The microscopical sphere fluctuates about its equilibrium position by thermal motion and therefore can be considered as a Brownian particle bound in a potential. The motion is readily described by the Langevin equation

$$
m \ddot{h}+\gamma(h) \dot{h}+\frac{\partial U(h)}{\partial h}=f_{\text {stoch }}
$$

Here $m \ddot{h}$ describes the acceleration, $\gamma(h) \dot{h}$ the hydrodynamic dissipative force, $U(h)$ the interaction potential, and $f_{\text {stoch }}$ the thermal noise. The hydrodynamic friction acting on the sphere is given by Stokes formula

$$
\gamma(h)=\Gamma(h) 6 \pi \eta R
$$

where $\Gamma(h)$ represents a dimensionless correction factor which accounts for the boundary condition imposed by the close proximity of the sphere to the plane wall. The factor $\Gamma(h)$ has been calculated analytically in the limit of slow viscous flow by Brenner. ${ }^{11}$ It can be shown that $\Gamma(h)$ $\approx R / h$ provided $h \ll R$. If we estimate $R \approx 10 \mu \mathrm{m}$ and $\langle h\rangle$ $\approx 100 \mathrm{~nm}, \Gamma(x)$ is typically of the order of 100 . Therefore the system is strongly overdamped and the acceleration term $m \ddot{h}$ in eq 5 may be truncated. We now calculate the autocorrelation function $\langle d h(t) d h(0)\rangle$ of the distance fluctuations $d h=h-\langle h\rangle$ making the following approximations. If the fluctuations $d h$ are small compared to $\langle h\rangle$, the position dependence of the friction is weak $\Gamma(h)$ $\approx \Gamma=R /\langle h\rangle$. Furthermore the potential can be harmonically approximated about its minimum with an effective force constant $U^{\prime \prime}\left(h_{0}\right)$. Then applying the dissipation fluctuation theorem, one obtains from eq 5 and eq 6

(11) Brenner, H. Chem. Eng. Sci. 1991, 16, 242.

$$
\langle d h(t) d h(0)\rangle \approx \frac{k_{\mathrm{B}} T}{U^{\prime \prime}\left(h_{0}\right)} e^{-t / \tau}
$$

where the relaxation time $\tau$ is given by

$$
\tau=\frac{6 \pi \eta R^{2}}{\langle h\rangle U^{\prime \prime}\left(h_{0}\right)}
$$

We now ask for the probability distribution function $p(h)$ of finding the sphere at a distance $h$. Since the whole system is in thermal equilibrium with its environment and no time-dependent external force is applied, the distribution function $p(h)$ must be Boltzmann distributed

$$
p(h)=A e^{-U(h) / k_{\mathrm{B}} T}
$$

where $U(h)$ denotes the potential of mean force. Therefore, provided the sphere is observed over a sufficiently long time, the interaction potential $U(h)$ is conversely given by the Boltzmann formula

$$
U(h) / k_{\mathrm{B}} T=-\ln P(h)+\text { constant }
$$

where $P(h)$ is the number of times that the sphere was found at a distance $h$. This equation provides a very direct method of determining the repulsive interaction potential between a sphere and the underlying substrate as has been noticed by Prieve et al. ${ }^{6}$

Interaction Potential. The interaction between latex beads or spherical vesicles and a glass surface can be described simply in terms of electrostatic, van der Waals, and gravitational forces.

According to the DLVO theory, ${ }^{12}$ the electrostatic double layer force per area for large distances $h>\kappa^{-1}$ is given by

$$
f_{\mathrm{el}}(h)=64 n k_{\mathrm{B}} T \tanh \frac{e \Psi_{1}}{4 k_{\mathrm{B}} T} \tanh \frac{e \Psi_{2}}{4 k_{\mathrm{B}} T} e^{-\kappa h}
$$

where $\kappa^{-1}$ denotes the Debey screening length, $n$ denotes the number density of ions, and $\Psi_{1}$ and $\Psi_{2}$ denote the surface potentials of the substrate and the sphere, respectively.

van der Waals forces do not contribute significantly to the long range forces measurable with the technique described above. However, in the case of extremely weak or short range repulsive forces, van der Waals forces are important and eventually cause adhesion of the particle to the substrate. For particles with finite wall thickness $d_{\mathrm{s}}$ the nonretarded van der Waals force per unit area is given by

$$
f_{\mathrm{VdW}}(h)=-\frac{A_{\mathrm{H}}}{6 \pi}\left(\frac{1}{h^{3}}-\frac{1}{\left(h+d_{\mathrm{g}}\right)^{3}}\right)
$$

where $A_{H}$ denotes the nonretarded Hamaker constant. Note that the effect of finite wall thickness $d_{\mathrm{s}}$ is relevant for vesicles or cells, while for latex spheres the second term vanishes $(d \rightarrow \infty)$.

The order of magnitude of the Hamaker constant in the long range regime ( $h>5 \mathrm{~nm}$ ) can easily be estimated according to the Lifshitz theory. Since dispersion forces are virtually cancelled by the retardation effect, only the zero frequency contribution is to be considered. (As a reference see e.g. the book by Israelachvili. ${ }^{13}$ ) The zero frequency contribution in water is approximately given by

(12) Verwey, E. J.; Overbeek, J. Th. Theory of the Stability of Lyophobic Colloids; Elsevier: Amsterdam, 1948.

(13) Israelachvilli, J. N. Intermolecular and Surface Forces; Academic Press: London, 1985. 


$$
A_{\mathrm{H}} \approx A_{\nu=0} \approx(3 / 4) k_{\mathrm{B}} T
$$

In electrolyte solution however the zero-frequency contribution is further reduced due to ionic screening ${ }^{14}$

$$
\begin{gathered}
A_{\nu=0}(h)=A_{\nu=0}(0) 2 \kappa h e^{-2 \kappa h} \\
\kappa h \gg 1
\end{gathered}
$$

The sum of the electrostatic and VdW forces per area, $f_{\mathrm{a}}$, has to be integrated over the surface of the sphere to obtain the actual force acting on the sphere. Using Derjaguins approximation ${ }^{15}$ the integration leads to

$$
F=2 \pi R \int f_{\mathrm{a}}(h) \mathrm{d} h
$$

Furthermore the gravitational force is given by

$$
F_{\mathrm{grav}}=\frac{4 \pi}{3} g \delta \rho R^{3}
$$

where $\delta \rho=\rho_{\mathrm{L}}-\rho_{\mathrm{W}}$ is the density difference between the sphere and water.

Then the total interaction potential $U(h)$ is finally otained by integration of the total force $F_{\text {total }}=F+F_{\mathrm{grav}}$

$$
\begin{array}{r}
U(h)=2 \pi R 64 n k_{\mathrm{B}} T \tanh \frac{\mathrm{e} \Psi_{1}}{4 k_{\mathrm{B}} T} \tanh \frac{e \Psi_{2}}{4 k_{\mathrm{B}} T} \kappa^{-2} e^{-\mathrm{kh}}- \\
\mathrm{R} \frac{A_{\mathrm{H}}}{6 h}+\frac{4 \pi}{3} g \delta \rho R^{3} h
\end{array}
$$

This theoretical potential can be directly compared with our experimental potential obtained from eq 10 . Clearly, the observation of distance fluctuations due to Brownian motion only provides information about the form of $U(h)$ near the potential minimum (corresponding to a potential difference $U(h)-U\left(h_{0}\right)$ of several $\left.k_{\mathrm{B}} T\right)$.

We now evaluate the mean distance $\langle h\rangle$, the mean square displacement $\left\langle d h^{2}\right\rangle$, and the relaxation time $\tau$ that are obtained from the autocorrelation function, eq 7 . For these quantities simple scaling relations for the dependence on the radius of the spheres hold. Firstly, the location of the potential minimum is easily shown from eq 17 to be

$$
\langle h\rangle \approx h_{0}=-\kappa^{-1} \ln R^{2}+\text { constant }
$$

provided that van der Waals forces can be ignored.

Secondly, by evaluating $U^{\prime \prime}\left(h_{0}\right)$, the mean square displacement $\left\langle d h^{2}\right\rangle$ is found to scale with the inverse of the third power of $R$

$$
\left\langle d h^{2}\right\rangle=\frac{k_{\mathrm{B}} T}{\kappa \frac{4 \pi}{3} \delta \rho g} \frac{1}{R^{3}}
$$

Finally from eq 7 and eq 8 it follows that the quantity $\tau\langle h\rangle /\left(d h^{2}\right\rangle$ is quadratic in $R$

$$
\frac{\tau\langle h\rangle}{\left\langle d h^{2}\right\rangle}=\frac{6 \pi \eta}{k_{\mathrm{B}} T} R^{2}
$$

It is important to realize that the right side of the last equation is purely determined by the hydrodynamic of the system and is independent of the potential, i.e. the ionic strength and the surface potential of the spheres. In other words the quotient of the relaxation time over the

(14) Mahanty, J.; Ninham, B. W. Dispersion Forces; Academic Press: New York, 1976.

(15) Derjaguin, B. Kolloid-Z, 1934, 69, 155.

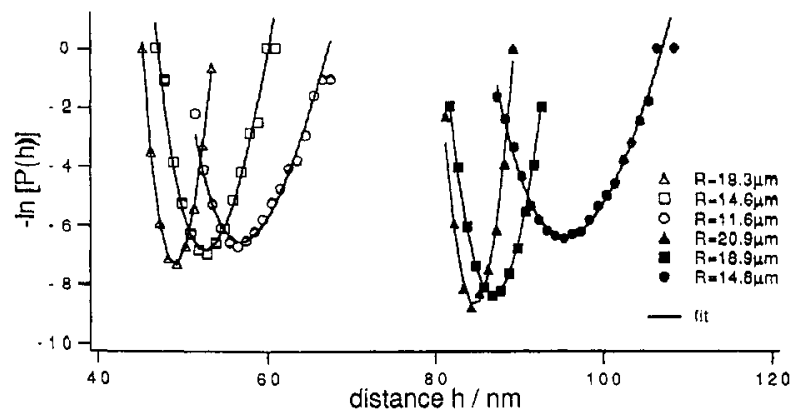

Figure 3. Negative logarithm of the distribution function of distances for spheres of different radii in $2 \mathrm{mM}(\Delta)$ and $0.5 \mathrm{mM}$ (A) $\mathrm{NaCl}$ solution. The plots indicate the interaction potential (in units of $k_{\mathrm{B}} T$ ) according to the Boltzmann relation.

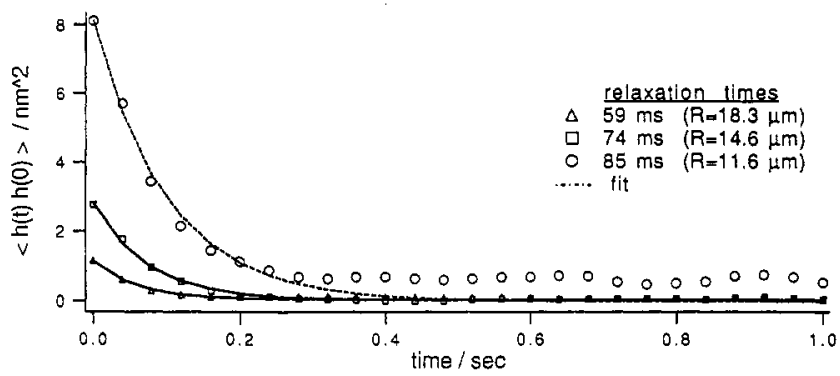

Figure 4. Time autocorrelation function of distance fluctuations of the spheres shown in Figure 5 in $2 \mathrm{mM} \mathrm{NaCl}$. The decay is single exponential.

mean square displacement is a direct measure for the dissipative force $\gamma(h)=k_{\mathrm{B}} T \tau /\left\langle d h^{2}\right\rangle$.

\section{Results}

We carried out several experiments with polystyrene spheres. Figure 3 shows the negative logarithms of the measured distribution functions $P(h)$ of six spheres of different radii in $\mathrm{NaCl}$ solution. It is obvious that with increasing size of the spheres the mean separation distance for large spheres shifts toward the glass surface. At the same time the mean square displacement decreases. Also, a lower salt concentration obviously enhances the range of electrostatic repulsion (compare results for 2 and 0.5 $\mathrm{mM} \mathrm{NaCl}$ in Figure 3). The solid lines were obtained by fitting of the experimental data to the theoretical potential given by eq 17 . The fitting procedure does not take account of the van der Waals interaction and leaves only the density and the surface potential of the sphere as free parameters. Assuming a surface potential of $70 \mathrm{mV}$ for glass, the densities of the spheres are found to lie between 0.06 and $0.09 \mathrm{~g} / \mathrm{cm}^{3}$ and the surface potential of the sphere lies between 20 and $60 \mathrm{mV}$.

It is noteworthy that the shape of the potential around its minimum is fairly symmetric, which justifies the harmonic approximation that was used to obtain the explicit form of the autocorrelation function eq 7 . The autocorrelation functions corresponding to the case of 2 $\mathrm{mM}$ ionic strength (Figure 3) are depicted in Figure 4. The plot includes best single exponential fits. At long correlation times in some cases deviations from the zero limit are found for autocorrelation function of smaller spheres. This effect might be due to convection.

The mean distance $\langle h\rangle$, the mean square displacement $\left\langle d h^{2}\right\rangle$, and the relaxation time $\tau$ have been determined for two sets of polystyrene spheres in two different ionic strengths in order to investigate the dependence of these parameters on the radius of the sphere. The experimental results are shown in Figure 5. Figure 5a depicts the dependence of the mean separation distance $\langle h\rangle$. The 

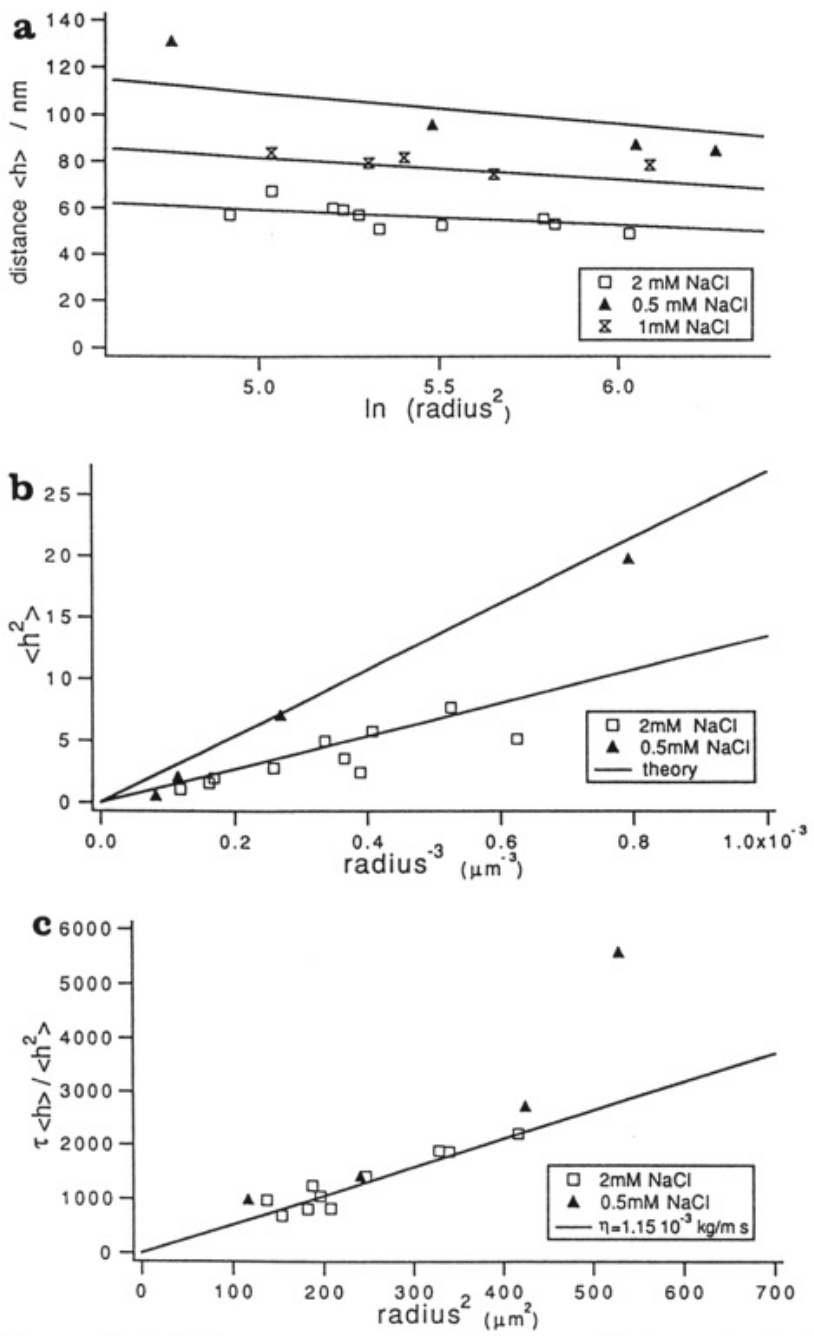

Figure 5. (a) The mean separation distance (sphere-glass) of latex beads of different size as a function of $\ln \left(R^{2}\right)$ for three different ionic strengths. The slope is equal to the Debey length $\kappa^{-1}$. (b) The mean square displacement as a function of $R^{3}$. (c) The enhanced dissipation of a sphere close to a wall is demonstrated by the fact that the dissipative force $k_{\mathrm{B}} T \tau /\left\langle d h^{2}\right\rangle$ is proportional to $R^{2} /\langle h\rangle$. The slope yields the viscosity of the medium.

theoretical lines have been calculated with eq 18 assuming $\delta \rho=0.065 \mathrm{~g} / \mathrm{cm}^{3}, \Psi_{\text {glass }}=70 \mathrm{mV}$, and $\Psi_{\text {latex }}=50 \mathrm{mV}$. The correct $\ln \left(R^{2}\right)$ dependence of $\langle h\rangle$ predicted by eq 18 is fullfilled. Note that the slope of the theoretical line is equal to the Debey length $\kappa^{-1}$, which is known from the molarity of the $\mathrm{NaCl}$ solution. Figure $5 \mathrm{~b}$ shows that $\left\langle d h^{2}\right\rangle$ scales with $R^{-3}$ as predicted by eq 19 . The experimental deviations are either real discrepancies in density and surface charge of the spheres or experimental errors in determinating the sphere-substrate distance. By repeated measurements on one single sphere we found $\langle h\rangle$ to be reproducible within $4 \mathrm{~nm}$.

In Figure $5 \mathrm{c}$ the dissipative hydrodynamic force is found to obey the $R^{2}$ dependence predicted from eq 20 . Therefore the modified Stokes formula ${ }^{11}$ for the hydrodynamic flow close to a wall correctly describes the slowing-down-effect of the wall. The straight line in this figure represents a linear fit that yields the viscosity of the medium $\eta=1.2$ $\times 10^{-3} \mathrm{~kg} / \mathrm{ms}$ directly from the slope of the line. The slightly too large value for the viscosity of water might be an effect of a relevant "hairiness" of the spheres. Single polystyrene chains stick out from the surface and cause a difference between the optically measured spheresubstrate distance and the hydrodynamic separation
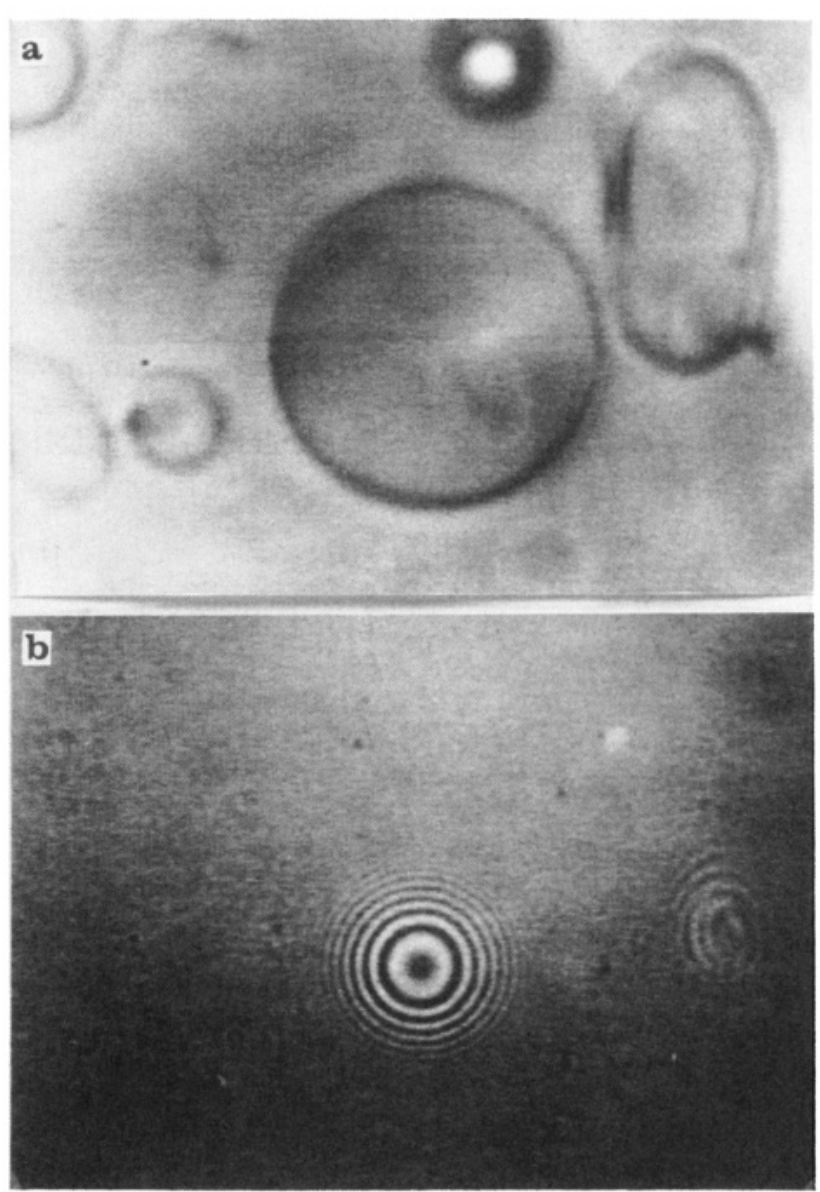

Figure 6. Phase contrast and RIC micrograph of a spherical DMPC vesicle $(R=8.3 \mu \mathrm{m})$ floating above a supported DMPC monolayer: (a) phasecontrast micrograph; (b) RIC micrograph. The spherical vesicle is under osmotic tension and shows no surface undulations.

distance in Stokes formula. An effective surface roughness of $5 \mathrm{~nm}$ compared to a separation distance of $50 \mathrm{~nm}$ could explain a $10 \%$ deviation in the viscosity.

Vesicle-Substrate Interaction. We present preliminary experiments on spherical giant lecithin (DMPC) vesicles interacting with a supported monolayer of DMPC on silanized glass. Hydrocarbon chains (octadecyltrichlorosilane) were chemically attached to a glass surface. Subsequently a monolayer was deposited by incubation with sonicated DMPC vesicles above the phase transition temperature. ${ }^{16}$ Giant DMPC vesicles were prepared in $180 \mathrm{mM}$ inositol solution. The experiment was carried out as follows. A certain amount of inositol/ $\mathrm{NaCl}$ solution was added to a giant vesicle suspension, such that the osmolarity of the solution decreases while at the same time the ionic strength was adjusted. The decrease in osmolarity leads to an increase of the volume of vesicles which eventually becomes spherical. Finally the vesicle suspension was filled into a microchamber with the bottom formed by a supported monolayer substrate.

Under the present preparation conditions one finds vesicles that are spherical and exhibit no visible surface undulations in either phase contrast or RIC microscopy (see Figure 6). However, the vesicles do not adhere but fluctuate above the DMPC substrate, as long as the ionic strength does not exceed $20 \mathrm{mM}$. This observation is surprising in the first place, because a neutral lipid like DMPC is expected to adhere by van der Waals forces.

(16) Merkel, R.; Sackmann, E.; Evans, E. J. Phys. (Paris) 1989, 50, 


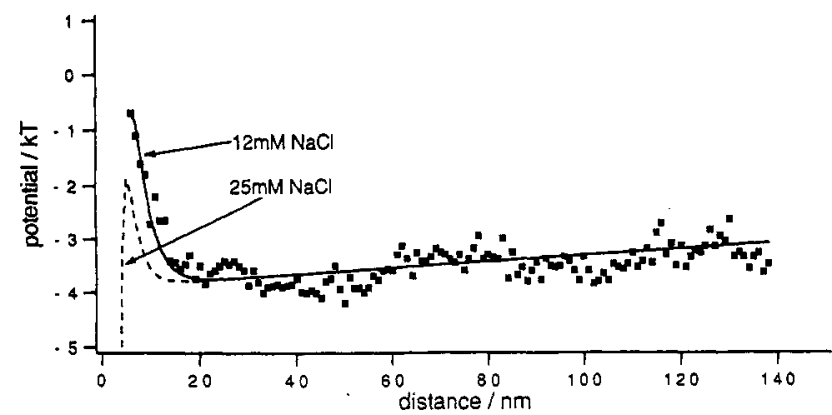

Figure 7. Interaction potential obtained from the distance fluctuations of a DMPC vesicle above a DMPC supported monolayer. The solid line depicts the theoretical interaction potential for $\Psi_{\text {monolayer }}=10 \mathrm{mV}, \Psi_{\text {vesicle }}=0.3 \mathrm{mV}$. Increasing the ionic strength to $25 \mathrm{mM}$ leads to adhesion due to van der Waals forces as indicated by the dashed line.

However, since the vesicles show distance fluctuations like rigid spheres, a corresponding repulsive potential can be determined as described above. Figure 7 depicts a measured interaction potential. The long range part of the potential exhibits a slightly linear increase due to gravitation. The corresponding density difference is found to be about $0.001 \mathrm{~g} / \mathrm{cm}^{3}$.

The repulsive force observed at $h<20 \mathrm{~nm}$ might be explained as an electrostatic effect. There are two possible reasons why the surfaces of the DMPC bilayer can be charged. Firstly, since glass surfaces are known to be strongly negatively charged $\left(\Psi_{\text {glass }} \approx 100 \mathrm{mV}\right)$, the supported DMPC layer might carry induced surface charges. Secondly, the addition of $\mathrm{NaCl}$ might effectively charge the vesicle due to different diffusion constants of sodium and chlorine ions through the vesicle membrane. Let us assume small surface charges on both surfaces, then the theoretical fit that includes electrostatic, van der Waals and gravitational forces according to eq $\mathbf{1 7}$ is shown in Figure 7. The fit yields a surface potential of $1.8 \mathrm{mV}$ for the symmetric case $\Psi_{1}=\Psi_{2}$. If one assumes a higher surface potential of the supported monolayer, say $\Psi_{1}$ and $10 \mathrm{mV}$, then $\Psi_{2}$ would be smaller, $\Psi_{2}=0.3 \mathrm{mV}$. A surface potential as low as $0.3 \mathrm{mV}$ corresponds to a charge density of 500 electrons $/ \mathrm{mm}^{2}$ or $0.05 \mathrm{~mol} \%$ charged lipid. Therefore impurities might also account for the observed extremely weak repulsion. Note that the VdW force has a stronger effect than it might seem from the experimental potential form. An increase in ionic stength to $25 \mathrm{mM}$ $\mathrm{NaCl}$ leads to adhesion due to $\mathrm{VdW}$ forces, as the theoretical dashed line indicates and as observed experimentally. Note that here only the screened zero-frequency contribution of the van der Waals force has been used, as described in the theoretical section.

\section{Conclusions}

We have demonstrated that the Brownian motion of a microsphere on a substrate can be followed and evaluated by fast image processing. The procedure yields the absolute distance dependence of the interaction potential about its minimum value and also the hydrodynamic frictional force of a spherical particle interacting with a wall. The interaction potential for latex spheres on glass is well-described by the electric double layer theory. The potential is fairly symmetric about the minimum and leads to simple scaling behavior of the mean distance, the mean square displacement, and the relaxation time. The hydrodynamic friction is found to be proportional to $R^{2} / h$ and yields the correct value for the viscosity of the medium. Therefore the presented technique is demonstrated to correctly measure long range forces of spherical objects interacting with defined substrates.

Our experiments on giant vesicles show that very weak forces can be measured. The range of measurable forces is determined by the mass of the investigated particles, i.e. the "restoring force" is gravitation. Thus for latex spheres the balancing forces are on the order of $10^{-11}-10^{-12}$ $\mathrm{N}$. For giant vesicles gravitation becomes extremely small, and forces as small as $10^{-15} \mathrm{~N}$ determine the distance fluctuations. This implies that very weak repulsive forces are detectable with the presented technique. A further application of the high sensitivity could be to investigate electrostatic solvent effects at bilayer surfaces. The technique is also noninvasive and can as well be applied to biological objects like red blood cells and any cells that can be made spherical. There are furthermore numerous applications arising from the fact that latex spheres can be easily chemically functionalized. Thus, for example, steric or specific receptor-ligand interactions can be investigated.

For obvious reasons the analysis of the Brownian motion yields information only about repulsive interaction forces. An essential refinement of the technique will be to apply an external force on the sphere. This would extend the measurable range of the interaction potentials and also would allow for adhesive forces to be measured. Here the application of latex spheres with magnetic core, variable substrate potentials, or optical tweezers might be possible future approaches.

Acknowledgment. We thank E. Evans, University of British Columbia, H. Risken, University of Ulm, Germany, and $H$. Versmold, University of Aachen, Germany, for helpful discussions. We are most grateful to Andreas Zilker for his help with the image processing system. This work was supported by the Deutsche Forschungs Gemeinschaft within the Sonderforschungsbereich project SFB 266 and by the Fond der Chemischen Industrie.

Registry No. DMPC, 13699-48-4. 